
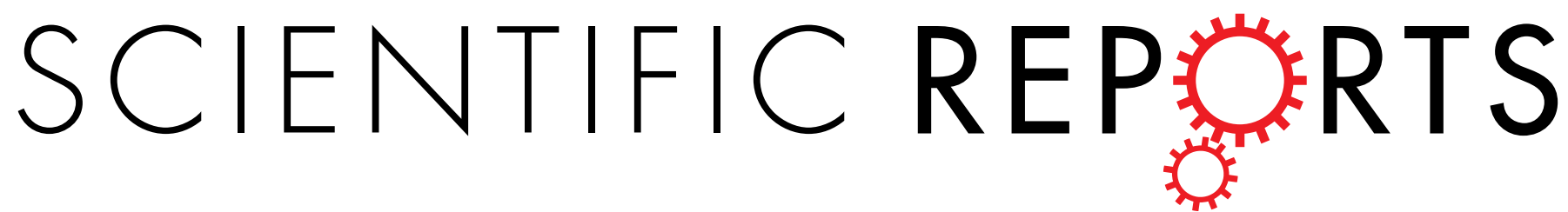

\title{
OPEN Binding of cellulose binding modules reveal differences between cellulose substrates
}

Received: 12 May 2016

Accepted: 28 September 2016

Published: 17 October 2016

\section{Suvi Arola ${ }^{1,2,3,+} \&$ Markus B. Linder ${ }^{2}$}

The interaction between cellulase enzymes and their substrates is of central importance to several technological and scientific challenges. Here we report that the binding of cellulose binding modules (CBM) from Trichoderma reesei cellulases Cel6A and Cel7A show a major difference in how they interact with substrates originating from wood compared to bacterial cellulose. We found that the CBM from $\operatorname{TrCel7A}$ recognizes the two substrates differently and as a consequence shows an unexpected way of binding. We show that the substrate has a large impact on the exchange rate of the studied CBM, and moreover, CBM-TrCel7A seems to have an additional mode of binding on wood derived cellulose but not on cellulose originating from bacterial source. This mode is not seen in double CBM (DCBM) constructs comprising both CBM-TrCel7A and CBM-TrCel6A. The linker length of DCBMs affects the binding properties, and slows down the exchange rates of the proteins and thus, can be used to analyze the differences between the single CBM. These results have impact on the cellulase research and offer new understanding on how these industrially relevant enzymes act.

Carbohydrate binding modules (CBMs) are found in a large variety of proteins that are active in one way or another on plant cell walls. These are mainly cellulases but also for example xylanases and mannanases ${ }^{1-4}$. There are several examples of convergent evolution leading to several different protein families with similar function ${ }^{5}$. Especially in bacteria these families are very diverse ${ }^{6}$. CBMs can show affinity for chitin and are also found as parts of chitin active proteins ${ }^{7}$. In this work we focus on the type of CBMs that are found in fungi and are classified as family ${ }^{1,2,8,9}$. These have been the subject of much investigation because of the efficiency and importance of fungal enzyme systems for degrading cellulosic material both as a part of the ecosystem and also increasingly for technical applications $\mathrm{s}^{10,11}$. These fungal CBMs have a compact structure comprising about 35 amino acids ${ }^{12}$. The structure has a stable cysteine knot fold that is also found in other small adhesion proteins such as conotoxins ${ }^{13}$. Cysteine knots are stabilized by disulfides and in the case of family 1 CBMs two or three of disulfide bridges are found. In the structure of the CBMs there is a distinctive arrangement of three aromatic residues that have been shown to interact with the cellulose surface. The interaction between aromatics and pyranose rings is widely observed also elsewhere, and it has been shown that by changing the character of the aromatic residues also the binding characteristics can be changed ${ }^{14}$. In addition to these pi-electron interactions, hydrogen bonding is involved in forming affinity and specificity between protein and cellulose $\mathrm{e}^{12}$. The binding is highly specific and shows even selectivity for the different crystalline faces of cellulose $\mathrm{e}^{15,16}$.

The CBMs have proved to play an essential role for how enzymes function. There are effects on both substrate recognition and catalytic activities ${ }^{16,17}$. The most straightforward explanation of CBM function is to guide the enzyme towards the substrate and increase local concentrations on surfaces, but in many cases the role has been found to be more subtle ${ }^{2}$. For example the exchange rate of cellulases from their substrates is greatly affected by the presence of a CBM ${ }^{17}$

In this work we set out to gain understanding of both cellulose as a substrate, and the functional role of CBMs in enzymes acting on cellulosic materials. It is already known that linking two CBMs together does affect the overall interaction of the proteins and substrate to a very large extent and that this two-domain interaction can to some extent explain the architecture of cellulose degrading enzymes in general ${ }^{7}$. The thermodynamic principles

${ }^{1}$ School of Science, Aalto University, P. O. Box 11100, FI-00076, Aalto, Finland. ${ }^{2}$ School of Chemical Technology, Aalto University, P.O. Box 16100, FI-00076, Aalto, Finland. ${ }^{3}$ VTT, Technical Research Centre of Finland, Bio and process technology, P.O.Box 1000, FIN-02044VTT, Finland. 'Present address: University of British Columbia, Michael Smith Laboratories, 2185 East Mall, Vancouver BC, V6T 1Z3, Canada. Correspondence and requests for materials should be addressed to M.B.L. (email: markus.linder@aalto.fi) 


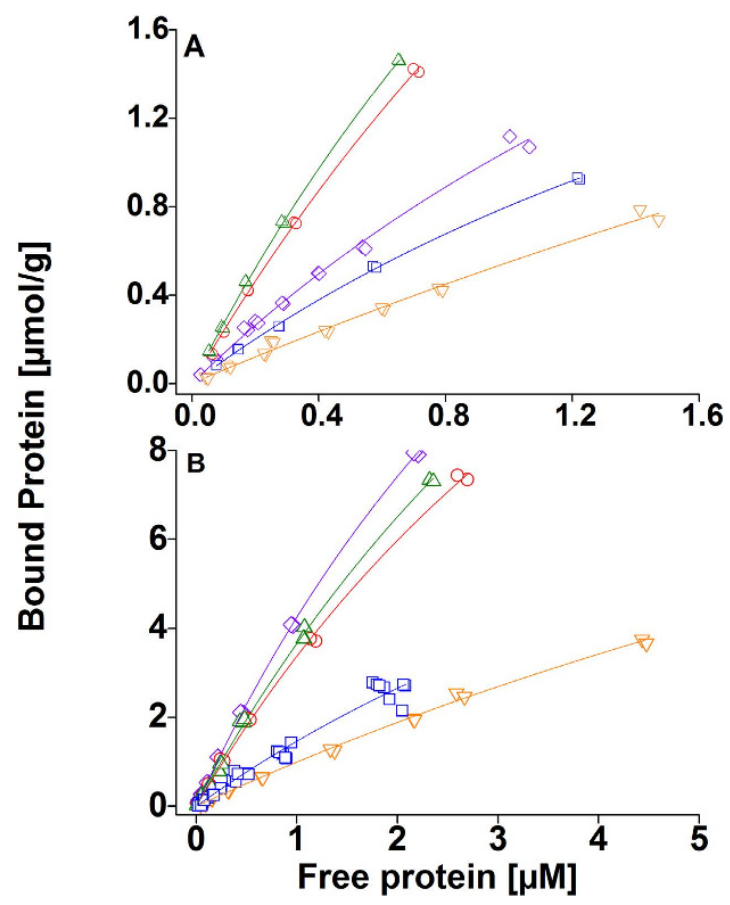

Figure 1. The initial slopes of the binding isotherms for CBM-Cel7A, CBM-Cel6A, DCBM-12, DCBM-24, and DCBM-48 on CNF and BMCC. (A) CBM-Cel7A and CBM-Cel6A, DCBM-12, DCBM-24, and DCBM-48 on BMCC, (B) CBM-Cel7A and CBM-Cel6A, DCBM-12, DCBM-24, and DCBM-48 on CNF. Violet diamond: CBM-CeI7A, orange triangle: CBM-Cel6A, red circle: DCBM-12, green triangle: DCBM-24, and blue square: DCBM-48.

behind such cooperativity are well known ${ }^{18}$ and depend on geometric constraints and the general architecture of the complexes that are formed. We hypothesized that making linkers with different lengths between two CBMs could reveal how binding sites are located on the cellulose surface and how the linking of binding modules changes the dynamics of interactions. Using a set of differently designed molecules, different sources of cellulose, and accurate measurement techniques enabling the study of binding kinetics allowed a new set of tools to investigate cellulose as complex structure and the structure-function relationship of the enzymes acting on it.

\section{Results}

Analysis of proteins. Double CBMs (DCBMs) with different linker lengths were produced as HFBI hydrophobin fusions (HFBI-DCBM) to aid purification (DCBM sequences shown in Supplementary Figure S1) as described previously ${ }^{19}$. The linkers were 12, 24 and 48 amino acids long and the proteins were named accordingly DCBM-12, -24 and -48 . The DCBM proteins were obtained by trypsin cleavage of the corresponding HFBIDCBM. The CBM-TrCel7A and CBM-TrCel6A were obtained by papain cleavage of HFBI-DCBM-12. Amino acid analysis (AAA) of the fractions from RP-HPLC run after papain cleavages showed the identity of the two fractions; i.e. which of them CBM-TrCel7A was and which CBM-TrCel6A.

Matrix-assisted laser desorption/ionization - time of flight mass spectrometry (MALDI-TOF MS) was used to verify the size of all the proteins and the extent of glycosylation of the linker regions. According to MALDI-TOF results DCBM-24 contained 14-26 glycan groups and DCBM-48 approximately 28-50 glycan groups. Both linkers (DCBM-24 and -48) contain multiple $O$-glycosylation sites (threonine (T) and serine (S), Supplementary Figure S1). The glycosylation patterns were heterogenic and showed a broad peak at $\mathrm{m} / \mathrm{z} \sim 12730-14500$ for DCBM-24 and $\mathrm{m} / \mathrm{z} \sim 18400-20730$ for DCBM-48. DCBM-12 did not show similar heterogenic glycosylation as DCBM-24 and -48 . DCBM-12 had a major peak at $\mathrm{m} / \mathrm{z} 9039$ and two minor peaks at $\mathrm{m} / \mathrm{z} 9265$ and 9446.

The single CBMs cleaved from HFBI-DCBM-12 by papain digestion did not show glycosylation in MALDI-TOF. The AAA results showed about $0,5 \%$ of glucosamine in the sample, which corresponds to 1 GlcNAc per 200 amino acids for both CBM samples. There were no peaks with differences by $\mathrm{m} / \mathrm{z} 162$ (hexose) or $\mathrm{m} / \mathrm{z} 203$ (GlcNAc). The main peak for CBM- $\operatorname{TrCel6A}$ was at $\mathrm{m} / \mathrm{z} 5414$ with minor peaks at $\mathrm{m} / \mathrm{z} 5015,5170,5214,1257$, $5313,5370,5501,5572,5659$, and 5743 . CBM- $\operatorname{TrCel} 7 \mathrm{~A}$ main peak was at $\mathrm{m} / \mathrm{z} 3850$ with minor peaks at $\mathrm{m} / \mathrm{z} 3763$, $3832,3952,4051$, and 4251 . For the major and minor peaks Na-adducts were seen.

Binding isotherms. We performed binding studies of all five CBM constructs with varying concentrations on two different substrates, namely cellulose nanofibrils (CNF) originating from hardwood and bacterial microcrystalline cellulose (BMCC). Figure 1 shows the initial slopes of the binding isotherms for CBMs and DCBMs on a) BMCC, and b) CNF. The corresponding partitioning coefficients, $\mathrm{K}_{\mathrm{r}}$, shown in Table 1 can be calculated from the dissociation constant, $K_{d}$, and the maximum binging capacities, $B_{\max }$, using equation (1), where $B$ is the amount of bound protein and Free is the amount of unbound protein. 


\begin{tabular}{|l|c|c|c|c|c|}
\hline & CBM-Cel7A & CBM-Cel6A & DCBM-12 & DCBM-24 & DCBM-48 \\
\hline BMCC & $1.40 \pm 0.46$ & $0.61 \pm 0.46$ & $2.47 \pm 0.55$ & $2.82 \pm 0.45$ & $1.07 \pm 0.15$ \\
\hline CNF & $4.98 \pm 0.56$ & $1.05 \pm 0.34$ & $3.87 \pm 0.75$ & $4.18 \pm 0.85$ & $1.67 \pm 1.12$ \\
\hline
\end{tabular}

Table 1. The partitioning coefficients $\left(\mathrm{K}_{\mathrm{r}}=\mathrm{B}_{\max } / \mathrm{k}_{\mathrm{d}}\right)$ for single and double CBM obtained from the Langmuir isotherm fitted to the data on Fig. $1 a, b$.

\begin{tabular}{|l|c|c|c|c|c|}
\hline & CBM-Cel7A & CBM-Cel6A & DCBM-12 & DCBM-24 & DCBM-48 \\
\hline $\mathrm{K}_{\mathrm{r}}^{\mathrm{CNF}} / \mathrm{K}_{\mathrm{r}}^{\mathrm{BMCC}}$ & 3.57 & 1.72 & 1.56 & 1.49 & 1.56 \\
\hline
\end{tabular}

Table 2. The correlations of partitioning coefficients, $K_{r}$, for single and double CBM between CNF and BMCC calculated from $\mathrm{K}_{\mathrm{r}}$-values in Table 1 .

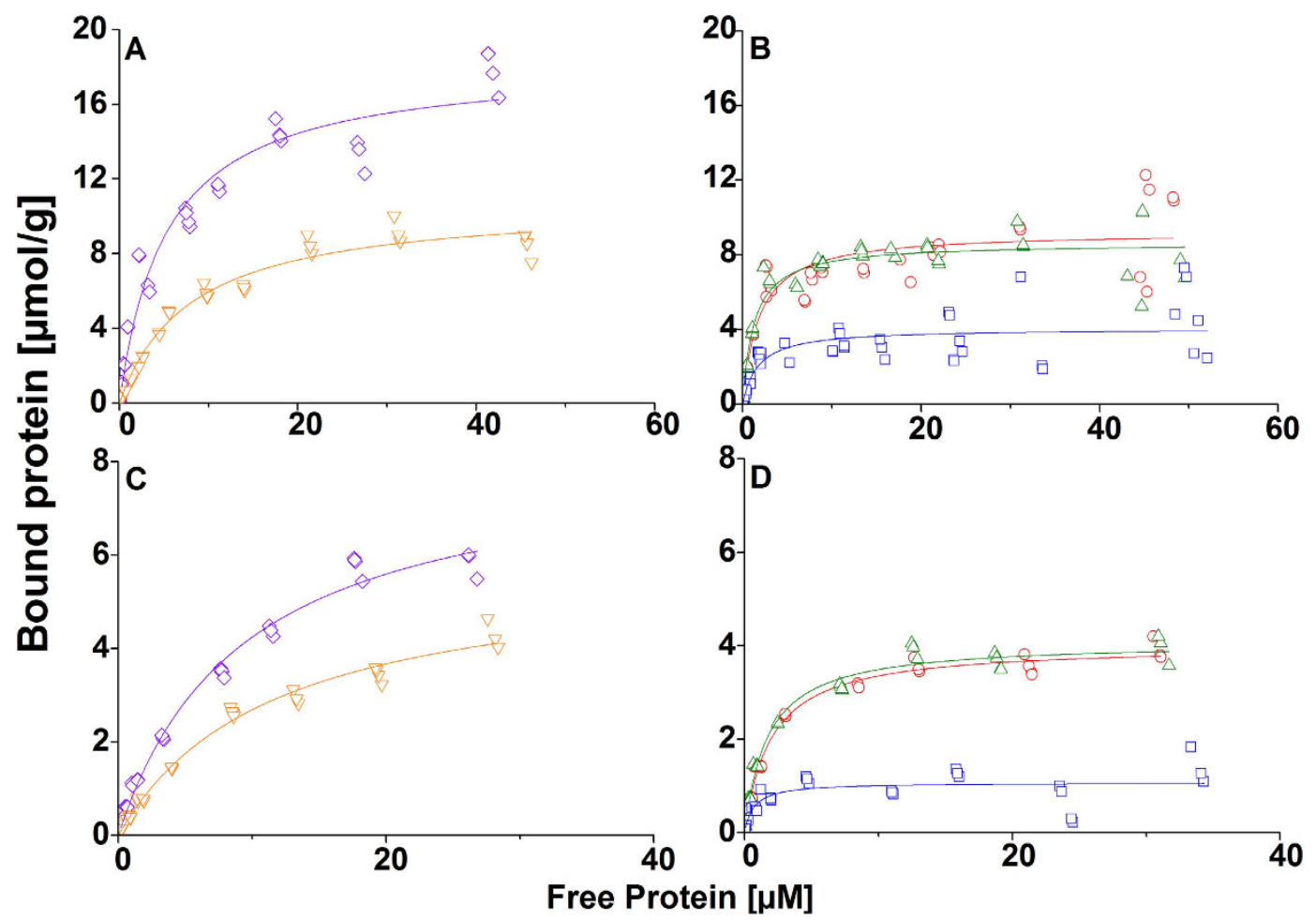

Figure 2. Binding isotherms for CBM-Cel7A, CBM-Cel6A, DCBM-12, DCBM-24, and DCBM-48 with high protein concentrations on CNF and BMCC. (A) CBM-Cel7A and CBM-Cel6A on CNF, (B) DCBM12, $-24,-48$ on CNF, (C) CBM-Cel7A and CBM-Cel6A on BMCC, and (D) DCBM-12, $-24,-48$ on BMCC. Violet diamond: CBM-CeI7A, orange triangle: CBM-Cel6A, red circle: DCBM-12, green triangle: DCBM-24, and blue square: DCBM-48.

$$
\lim _{\text {Free } \rightarrow 0}\left(\frac{d B}{d \text { Free }}\right)=\frac{\left[B_{\text {max }}\right]}{K_{d}}=K_{r}
$$

The $\mathrm{K}_{\mathrm{d}}$ and and $\mathrm{B}_{\max }$ values obtained from curve-fitting and used to calculate the $\mathrm{K}_{\mathrm{r}}$-values are listed in Supplementary Table S5. On BMCC DCBM-12 and DCBM-24 constructs have a significantly higher $\mathrm{K}_{\mathrm{r}}$-value compared to single CBMs and DCBM-48. On CNF the $\mathrm{K}_{\mathrm{r}}$-values for the DCBMs followed the same order as on BMCC; $\mathrm{K}_{\mathrm{r}}(\mathrm{DCBM}-24)>\mathrm{K}_{\mathrm{r}}(\mathrm{DCBM}-12)>\mathrm{K}_{\mathrm{r}}(\mathrm{DCBM}-48)$. The $\mathrm{K}_{\mathrm{r}}$-value of CBM-TrCel7A on CNF was higher than for any other protein and the $\mathrm{K}_{\mathrm{r}}$ for CBM-TrCel6A was the lowest.

To compare binding on the two substrates, the ratio of $\mathrm{K}_{\mathrm{r}}$-values $\left(\mathrm{K}_{\mathrm{r}}^{\mathrm{CNF}} / \mathrm{K}_{\mathrm{r}}^{\mathrm{BMCC}}\right)$ was calculated (Table 2). This ratio was the same for all other proteins, (about 1.5), except for CBM-TrCel7A for which it was 3.6.

Figure 2 presents the binding isotherms of all five proteins for both substrates with large protein concentrations (up to $50 \mu \mathrm{M}$ free protein concentration). The semi-logarithmic plots of the same data are presented in Supplementary Figure S2 to illustrate the saturation of the binding. The $\mathrm{B}_{\max }$ obtained from these data are presented in Table 3. 


\begin{tabular}{|l|c|c|c|c|c|}
\hline & CBM-Cel7A & CBM-Cel6A & DCBM-12 & DCBM-24 & DCBM-48 \\
\hline BMCC & $8.20 \pm 0.30$ & $5.78 \pm 0.21$ & $4.01 \pm 0.07$ & $4.09 \pm 0.08$ & $1.07 \pm 0.08$ \\
\hline CNF & $18.24 \pm 0.66$ & $10.74 \pm 0.37$ & $9.21 \pm 0.34$ & $8.61 \pm 0.21$ & $4.05 \pm 0.23$ \\
\hline
\end{tabular}

Table 3. Binding capacities, $B_{\max }\left(\mu \mathrm{molg}^{-1}\right)$, for single and double CBM on CNF and BMCC obtained from the data shown in Fig. 2.

\begin{tabular}{|l|c|c|c|c|c|c|}
\hline substrate & B $_{\max }{ }^{*}$ & CBM-Cel7A & CBM-Cel6A & DCBM-12 & DCBM-24 & DCBM-48 \\
\hline \multirow{3}{*}{ CNF } & low & -30.7 & -28.2 & -31.8 & -32.1 & \multirow{2}{*}{-31.7} \\
\cline { 2 - 6 } & inter & -29.0 & -26.5 & -30.1 & -30.4 & \\
\cline { 2 - 6 } & high & -28.0 & -25.5 & -29.1 & -29.4 & \\
\hline \multirow{3}{*}{ BMCC } & low & -29.6 & -28.4 & -32.7 & -33.0 & \multirow{2}{*}{-33.9} \\
\cline { 2 - 6 } & inter & -27.9 & -26.7 & -31.0 & -31.3 & \\
\cline { 2 - 6 } & high & -26.9 & -25.7 & -30.0 & -30.3 & \\
\hline
\end{tabular}

Table 4. Gibbs free energy for the binding, $\Delta \mathrm{G}(\mathrm{kJ} / \mathrm{mol})$, for CBM-Cel7A, CBM-Cel6A, DCBM-12, DCBM-24, and DCBM-48 on CNF and BMCC. ${ }^{*} B_{\max }-$ value for DCBM-48 from data in Fig. 2 is an accurate value, thus it can be used to calculate the binding energy associated with the binding event. The $\Delta \mathrm{G}$-values for CBM-Cel7A, CBM-Cel6A, DCBM-12 and DCBM-24 are estimates (see supplementary information for the estimation, Supplementary Fig. 3). $B_{\text {max,low }}$ is the value gained from data on Fig. 2 , inter is $2 x\left(B_{\text {max,low }}\right)$, and high is $3 \mathrm{x}\left(\mathrm{B}_{\mathrm{max}, \text { low }}\right)$.

The Gibbs free energy of binding, $\Delta \mathbf{G}$. In order to identify differences in the binding energies of the proteins that could account for the different behavior of CBM-TrCel7A on CNF, we calculated the $\Delta \mathrm{G}$-values for all five proteins on both substrates using the $B_{\max }$-values obtained from the data presented in Fig. 2 (Supplementary Figure S2) and the $\mathrm{K}_{\mathrm{r}}$-values presented in Table 1 using equation (2).

$$
\Delta G=R T\left(\ln \left(B_{\max }\right)-\ln \left(K_{r}\right)\right)
$$

For the binding of DCBM-48 on both substrates the maximum binding capacity was clearly reached (Fig. 2b,d and Supplementary Figure S2b,d). Thus, the $\mathrm{B}_{\max }$-values could be used as such for the $\Delta \mathrm{G}$ calculations. For the other proteins the actual $B_{\max }$ was not reached in our experiments (Fig. 2 and Supplementary Figure S2). In order to calculate an estimated range of the $\Delta \mathrm{G}$-values for these proteins, we used the $\mathrm{K}_{\mathrm{r}}$-values (Table 1) and a range of the $B_{\max }$-values in equation (2). The $B_{\max }$-value range used was $B_{\max }$ low, $B_{\max }$, intermediate, and $B_{\max }$, high. A schematic illustration of the three $B_{\max }$-values are presented in Supplementary Figure S3 with the binding isotherm for CBM-TrCel7A on CNF. Table 4 summarizes the $\Delta \mathrm{G}$-values for the different proteins on both substrates.

Exchange rates of CBMs and DCBMs on CNF and BMCC. Next, to investigate the exchange of DCBMs and CBMs on both substrate surfaces, we performed a series of experiments measuring the exchange rate at steady-state between ${ }^{3} \mathrm{H}$-labelled proteins and unlabeled proteins. The results presented in Fig. 3a show that exchange between adsorbed and free CBM-TrCel7A and CBM-TrCel6A readily occurs on BMCC surface, reaching $50 \%$ of labelled protein on the surface from the original amount. The rates of exchange are fast. The exchange occurs during tens to hundreds of seconds, which is in line with previous results gained for CBM- $\operatorname{TrCel} 7 \mathrm{~A}$ on $\mathrm{BMCC}^{20}$. Only partial exchange was detected in the case of DCBMs on BMCC; 10\% of DCBM-12 and -24 , and $\sim 30 \%$ of DCBM-48 exchange (Fig. 3b). Approximately $20-25 \%$ of the single CBMs (Fig. 3c) and $5-10 \%$ of the DCBMs (Fig. 3d) exchange on the CNF surface.

Nanoscale effect of the CNF substrate. CNF is much finer in structure due to its nanoscale scale size than commonly used substrates for cellulase studies such as microcrystallinen cellulose (MCC) or BMCC. To test if the nanoscale structure of the substrate affect the binding properties of the proteins we performed binding studies on the pulp material that the CNF was prepared from. The initial slopes of the binding isotherms and the corresponding $\mathrm{K}_{\mathrm{r}}$-values are shown in Supplementary Figure S4. The order of binding on pulp was the same as for CNF; CBM-TrCel7A has the steepest initial slope and thus the largest partitioning coefficient followed by the DCBMs and CBM-TrCel6A. The $\mathrm{K}_{\mathrm{r}}$-values are very close to those obtained for CNF and thus the binding mode or mechanism seems to be the same as for CNF.

Xylanase assay for CBM-TrCel7A. The CNF that was used for the experiments was found to contain roughly $27 \%$ xylan by total enzymatic hydrolysis and sugar content analysis. Of this, $30 \%$ can be specifically hydrolyzed by pI9 xylanase from $T$. reese $i$, which corresponds to about $10 \%$ loss of the total mass ${ }^{21}$. To test how the xylan on CNF affects the CBM-TrCel7A binding we used the pI9 xylanase to hydrolyze xylan from CNF and tested how the treatment affected the binding of CBM-TrCel7A on CNF. We used a fixed concentration of protein and xylanase treated CNF, and quantified the amount of free protein from the reaction mixture using tritium labeled CBM-TrCel7A. As a control for the experiment we used CNF that was not treated with xylanase. All reactions were carried out in three replicates. The results showed that the binding of CBM- $\operatorname{TrCel7A}$ increased about $20 \%$ on the xylanase treated CNF compared to the non-treated CNF. 


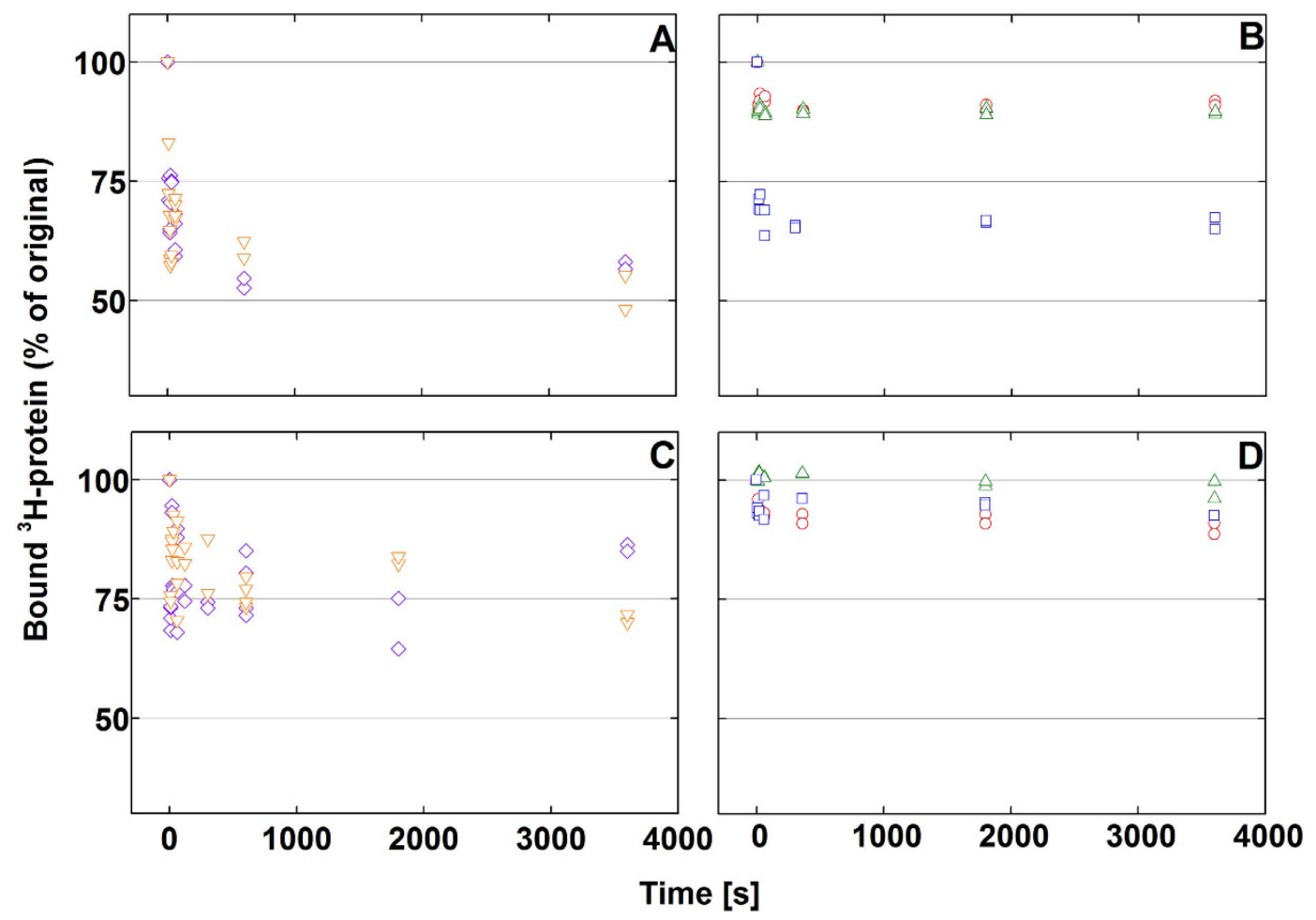

Figure 3. The exchange of ${ }^{3} \mathrm{H}$-labelled proteins from substrate surface with the non-labelled protein in the solution during time. (A) Exchange of ${ }^{3} \mathrm{H}$-labelled CBM-Cel7A and CBM-Cel6A from BMCC surface, (B) Exchange of ${ }^{3} \mathrm{H}$-labelled DCBM-12, DCBM-24, and DCBM-48 from BMCC surface, (C) Exchange of ${ }^{3} \mathrm{H}$-labelled CBM-Cel7A and CBM-Cel6A from CNF surface, and (D) Exchange of ${ }^{3} \mathrm{H}$-labelled DCBM-12, DCBM-24, and DCBM-48 from CNF surface. Violet diamond: CBM-CeI7A, orange triangle: CBM-Cel6A, red circle: DCBM-12, green triangle: DCBM-24, and blue square: DCBM-48.

\section{Discussion}

The results of this study show that the linker length of the DCBMs had a large effect on their binding properties and that the binding properties are greatly affected by the origin of the cellulose substrate. Surprisingly, we found out that CBM-TrCel7A, had a much higher partitioning coefficient on wood cellulose compared to the other proteins (Table 1), although, based on previous studies ${ }^{7}$ and general knowledge on polyvalent interactions ${ }^{18}$ we anticipated that the DCBMs would have a higher affinity towards cellulosic substrates than the single CBMs. We also saw that in addition to effects on the binding properties the linking of the CBMs together slowed down the exchange of bound protein from the substrate surface regardless of the substrate origin (Fig. 3b,d). However, the substrate origin had an effect on the exchange of the single CBMs (Fig. 3a,c).

The $\mathrm{K}_{\mathrm{r}}$-values allow comparison of the binding efficiency of the different proteins. But to investigate the difference of protein binding between substrates, BMCC and CNF, we calculated the ratio of $\mathrm{K}_{\mathrm{r}}$-values for each protein on the two different substrates (Table 2). The ratios of the partitioning coefficients for the DCBMs and CBM-TrCel6A were about 1.5. This result indicates that these proteins bind to the two substrates in a similar manner, except that CNF has 1.5 times more surface area per weight than BMCC. CBM- $\operatorname{TrCel7A}$ on the other hand had an overall higher $\mathrm{K}_{\mathrm{r}}$-value on CNF compared to the other proteins, and the ratio of $\mathrm{K}_{\mathrm{r}}$-values was over two times higher than for the other constructs. From these results we can deduce that the CBM- $\operatorname{TrCel7A}$ has another binding mode or site on CNF that it does not have on BMCC, and which the CBM-TrCel6A or the DCBMs do not have. Some feature in CNF is available for CBM-TrCel7A that does not exist in BMCC, yet this feature is not available for the other constructs. It is especially notable that the synergies between modules seen for the DCBM-12 and DCBM-24 when binding to BMCC are not seen when the proteins bind to CNF. DCBM-12 and DCBM-24 are thus not able to incorporate a possible extra mode of binding (e.g. conformational changes) or binding site of CBM- $\operatorname{TrCel7A}$ when they bind to CNF, yielding lower $\mathrm{K}_{\mathrm{r}}$-values compared to CBM- $\operatorname{TrCel7A}$.

The linker length had an effect on the binding properties of the DCBMs following the same order on both substrates; $\mathrm{K}_{\mathrm{r}}(\mathrm{DCBM}-24)>\mathrm{K}_{\mathrm{r}}(\mathrm{DCBM}-12)>\mathrm{K}_{\mathrm{r}}(\mathrm{DCBM}-48)$. These results indicate that there is an optimum linker length for the DCBMs in respect to maximum binding affinity, close to or equal to 24 amino acids. The positive effect on the affinity of multivalent interactions is well known ${ }^{18}$. In our case, however, it seems to be substrate dependent behavior, which is dictated by unexpected binding characteristics of one of the binding units.

To investigate if the overall higher affinity of CBM- TrCel7A towards CNF would be caused by a more favorable binding energy we estimated the $\Delta \mathrm{G}$-values for the different binding events. In order to conduct this estimation, we first experimentally determined reliable values for maximum binding capacities, $B_{\max }$, because the uncertainty of the $\mathrm{B}_{\max }$ (and hence the $\mathrm{k}_{\mathrm{d}}$ ) is large when only using initial parts of the isotherms for fitting. Thus, we evaluated if measuring full binding isotherms, allowing the calculation of binding energies, would be feasible. 
The uncertainty of $B_{\max }$-values is clearly seen by the differences of the estimated values shown in Table S5 compared to the actual measured values shown in Table 3. Data points were collected at very high protein concentrations (around $50 \mu \mathrm{M})$. At these concentrations only about $1-10 \%(\mathrm{DCBM})$ or $20 \%(\mathrm{CBM})$ of the total protein are bound to cellulose. Due to the high overall protein concentrations and low percentage of total protein bound to cellulose it was not feasible to acquire data points at even higher concentrations. A small error (1-2\%) in measuring the free protein concentration would result in a large error in bound protein amount (40-50\%). The data points are shown in Fig. 2, and as semi-logarithmic plots in Supplementary Figure S2 to illustrate how close to saturation the binding curves are. The $\mathrm{B}_{\max }$-values obtained from the full binding isotherms are presented in Table 3. From the data it is seen that the DCBM- 48 has reached its maximum binding capacity on both substrates, and that the DCBM-12 and -24 are very close to reaching their actual maximum binding capacity. Using the structure of CBM-Cel7a determined by Kraulis et al. ${ }^{22}$ we can estimate that one CBM requires at least $1.8 \mathrm{~nm}$ $\star 3 \mathrm{~nm}$ surface area of cellulose to bind, that is $5.4 \mathrm{~nm}^{2}$. This gives a lower limit for the available surface area to bind of $59 \mathrm{~m}^{2} / \mathrm{g}$ and $27 \mathrm{~m}^{2} / \mathrm{g}$ for CNF and BMCC, respectively. The $\mathrm{B}_{\max }$-values for DCBM- 48 are lower on both substrates compared to the $B_{\max }$-values of DCBM-12 and DCBM-24. The single CBMs have generally higher $\mathrm{B}_{\max }$-values on both substrates compared to the DCBMs, and they have not reached the actual maximum binding capacities. This too low $\mathrm{B}_{\max }$ can lead to an underestimation of $\Delta \mathrm{G}$. The natural linker of $\operatorname{TrCel7A}$ enzyme is 27 amino acids long ${ }^{23}$ and that of $\operatorname{TrCel6A}$ enzyme is 51 amino acids long ${ }^{24}$. The linker length clearly affects both the binding affinity and the binding capacity of the DCBMs and can thus also be a crucial element in controlling the binding affinity and capacity of the cellulases in nature. Igarashi et al. have shown that if the packing density of the enzymes on the substrate surface is too high the processivity of the cellulase is hindered ${ }^{16}$. In this respect the double binding module structure of the cellulase could be beneficial not only by concentrating or locating the enzyme on the substrate surface ${ }^{25}$ but also by controlling the amount of enzyme on the substrate surface, and in this way contributing to the hydrolysis efficiency of the enzymes.

The $\Delta \mathrm{G}$-values, presented in Table 4 , show that the binding energies for DCBMs are generally more negative than those for CBMs ( $\sim-16 \%$ on CNF and $\sim 10-17 \%$ on BMCC), and thus show that for all DCBMs there is a synergy in binding that comes from the linkage. In this regard it is energetically favorable to link CBMs together as DCBMs. It is also logical when considering the action of cellulase enzymes. However, these results do not explain the higher affinity of CBM-Cel7A on CNF.

Next we studied the exchange of free and bound protein on both substrates by equilibrium exchange experiments. On BMCC both CBMs alone fully exchanged to $50 \%$ of the original amount rather quickly $(<600 \mathrm{~s})$ and thus showing true reversibility (Fig. 3a) as previously shown for CBM-TrCel7A on BMCC ${ }^{26}$. On CNF, the single CBMs did not exchange to $50 \%$ from the original amount of bound, but only to $20-25 \%$ (Fig. $3 \mathrm{c}$ ) showing that the CBMs experience the two substrates differently.

The DCBMs showed only partial exchange from BMCC (Fig. 3b) and CNF (Fig. 3d), 10-30\% from BMCC and $5-10 \%$ from CNF. This partial exchange has been previously seen for HFBI-DCBM on CNF ${ }^{27}$. In nature slow processive enzymes such as cellulases could benefit from this reduced amount of protein exchange caused by the linking of two affinity proteins allowing more time for the hydrolysis reaction. It has been shown with high speed atomic force microscopy that $\operatorname{TrCel7A}$ cellulase carrying a mutation in its catalytic domain preventing its binding to the substrate surface and TrCel7A lacking the CBM have a faster exchange rate and lower activity than the wild type enzyme ${ }^{17}$. In this respect a double affinity module with reduced exchange from the substrate surface seem to be more effective in cellulose hydrolysis than a single affinity module with higher and potentially fully reversible exchange.

To investigate if mechanical processing of pulp to CNF had an effect on the substrate, we determined the initial slope of the binding isotherm on the pulp material used to make the CNF. The partitioning coefficients of the proteins on pulp were very close to those obtained for CNF and that the order of binding was the same (Supplementary Figure S4) showing that CNF processing was not the reason for our results.

We next considered if differences in polysaccharide composition between the two substrates that could explain affect results. BMCC is pure cellulose whereas CNF form birch has, in addition to cellulose, roughly $27 \% \mathrm{xylan}^{21}$. To try and understand the difference in binding sites available for CBM-TrCel7A on CNF but not on BMCC, we investigated how the specific hydrolysis of xylan affected the binding. This was done by using a xylanase enzyme to hydrolyze xylan from CNF and to study the binding of CBM-TrCel7A after the treatment in comparison to non-treated CNF. We saw that the amount of bound CBM-TrCel7A was increased by approximately $20 \%$ after xylanase treatment. From previous studies we know that the xylanase treatment reduces the amount of xylan in CNF by roughly $30 \%{ }^{21}$. This indicates that the CBM-TrCel7A finds more binding sites on the fibril surface after xylanase treatment due to an increase in the amount of exposed cellulose surface. Thus, the higher affinity of CBM-TrCel7A towards CNF would not be caused by an additional affinity towards xylan that is present in the CNF material. It has also been shown with cellulases, that xylanase enzymes are needed in order for the cellulases to fully hydrolyze CNF and that cellulases alone are not enough to hydrolyze materials that contain hemicellu$\operatorname{loses}^{28}$. We showed here that the binding of CBM-TrCel7A is positively affected by xylan hydrolysis and that at least partly the xylan available for the xylanase is located on the surface of the fibril covering the CBM- $\operatorname{TrCel} 7 \mathrm{~A}$ binding sites.

It now seemed evident that the differences in the substrates that are most obvious were not fully explaining the unexpected behavior of CBM-TrCel7A upon binding to CNF. The features, which are origin dependent in cellulose substrates are their crystalline structure and amorphous to crystalline ratio; wood cellulose crystalline regions compose mainly of cellulose $\mathrm{I}_{\beta}$ and BMCC of cellulose $\mathrm{I}_{\alpha}$, and BMCC is more crystalline ${ }^{29-31}$. Finding a way to test the effects of the crystalline structure on the binding properties is tricky, and some methods to analyze the morphological targets of CBMs have been devoleped ${ }^{25}$, but by competition experiments we could see if the two CBMs share binding sites on the two substrates. This on the other hand might reveal differences on the substrate structures. To test if the two CBMs fully or partially compete on binding sites on CNF and BMCC we 
designed a set of experiments where we tried to see if the binding of the one CBM is affected by the presence of the other. This was examined by competing one labelled CBM with another unlabeled CBM (Experimental in Supplementary Information, data in Supplementary Figure S6). The results showed that the binding of both labeled CBMs were affected by the presence of the unlabeled different CBM in the same way as they were affected by the presence of the unlabeled identical CBM. This suggests that the two CBMs would fully compete on binding sites on both substrates, and that the deviant binding behavior of CBM-TrCel7A on CNF would be caused by an extra mode of binding rather than an extra binding site recognized only by CBM- $\operatorname{TrCel} 7 \mathrm{~A}$.

The more efficient binding of the CBM-TrCel7A on CNF could also be caused by $O$-mannosylation of the binding module near the binding site ${ }^{32,33}$. Our proteins were produced in T. reesei, and the introduction of $O$-glycans could be possible. Yet, we saw no evidence of mannosylation of the CBMs with MALDI-TOF MS and AAA. The main peak of CBM- $\operatorname{TrCel7A}$ at $\mathrm{m} / \mathrm{z} 3850$ was very close to the average molecular mass obtained by AAA $(3828 \mathrm{Da})$ and to the theoretical molar mass of the CBM-TrCel7A calculated from the amino acid sequence $(3828 \mathrm{Da})$. None of the minor peaks matched with hexoses or HexNAc. The AAA results showed only little variation in the amounts of individual amino acids (aa) and the variation was concentrated on the amino acids present in the linker; namely serine and threonine. There were some traces of glucoseamine in the sample $(\sim 0.5$ mol-\%, meaning 1 sugar molecule per 200 amino acids) but this was too low to account for protein glycosylation. Similar findings were made for CBM-TrCel6A with AAA. However, more variation was seen in the linker region aa amounts compared to $\mathrm{CBM}-\operatorname{Tr} \mathrm{Cel} 7 \mathrm{~A}$. This indicates that the linker regions present on both sides of the CBM-TrCel6A show some heterogeneity due to papain cleavage. In contrast, the CBM-TrCel7A has only $\mathrm{N}$-terminal flanking linker. There were similar amounts of glucosamine present in the CBM-TrCel6A sample as was in the CBM-TrCel7A sample indicating a minor co-purification with the protein, rather than glycosylation. Furthermore, the mass differences in MALDI-TOF MS did not match glycan groups. Our results from AAA and MS data concluded that the single CBMs were not glycosylated, and that the variation of masses with in the samples arouse from the variation in the lengths of the flanking linker regions.

Conclusions. The work reveals that there is a difference in the way in which CBM-Cel7A interacts with cellulose of different origin compared to CBM-Cel6A. The CBM therefore has a direct effect on the substrate recognition of cellulase and not only on as previously reported, on the location of binding on the cellulose crystal. The results also show that BMCC may not be an ideal substrate for cellulase structure-function studies if the aim is to understand how plant cell walls are degraded. The results also show that substrate recognition is affected by the way in which CBMs are linked. The linkage of CBM-Cel6A to CBM-Cel7A in the DCBD did not give coupled binding energies nor exchange rates in the same way on CNF as on BMCC. This study shows that the CBM-substrate interaction is more complex than previously predicted and highlights in a new way that the choice of model substrates can affect outcomes of cellulase structure function studies.

\section{Methods}

Cellulose substrates. Cellulose nanofbrils (CNF) were prepared by fluidizing never-dried bleached Birch pulp (kappa number 1; DP 4,700; fines removed (SCAN-M 6:69)). The pulp was washed to the sodium form according to Swerin et al. ${ }^{3}$. The washed pulp was disintegrated with a high-pressure fluidizer (Microfluidics M110P, Microfluidics Int. Co., Newton, MA) in 12 passes essentially according to previous reports ${ }^{31}$. No chemical or enzymatic pre-treatment was used prior to disintegration. Bacterial microcrystalline cellulose (BMCC) was prepared from Nata de Coco by several washing and grinding steps.

Protein production and purification. The DCBMs were produced as HFBI-DCBM fusions in T. reesei as described in details in earlier work of the group ${ }^{19}$ (DCBM sequences in Supplementary Figure S1) and the following transformants were used: VTT-D-133335 (HFBI-DCBM-12), VTT-D-133336 (HFBI-DCBM-24), and VTT-D133337 (HFBI-DCBM-48). The strains were then cultivated in $7 \mathrm{~L}$ bioreactors on media containing 50 vol- $\%$ spent grain extract, $60 \mathrm{~g} / \mathrm{L}$ lactose, 1 g/L yeast extract, 4 g/L KH2PO4, 2.8 g/L (NH4)2SO4, 0.6 g/L MgSO 4 . 7H2O, $0.8 \mathrm{~g} / \mathrm{L} \mathrm{CaCl} 2 \cdot 2 \mathrm{H} 2 \mathrm{O}, 2 \mathrm{ml} / \mathrm{L}$ trace solution. The $\mathrm{pH}$ was let to drop from 5 to about 3 during cultivation. At $24 \mathrm{~h}$ intervals $48 \mathrm{mg}$ pepstatin A and $28 \mathrm{mg}$ soy bean trypsin inhibitors (both from Sigma-Aldrich) were added to the cultures to minimize protein degradation. The culture supernatants were separated from the biomass by filtration through GF/A filters (Whatman). Protein expression levels were analyzed by RP-UPLC and were $0.2 \mathrm{~g} / \mathrm{L}, 0.4 \mathrm{~g} / \mathrm{L}$, and $3.0 \mathrm{~g} / \mathrm{L}$ for HFBI-DCBM-12, -24 , and -48 , respectively. The proteins were purified using aqueous two phase extraction and reverse-phase high-performance liquid chromatography (RP-HPLC) as described earlier ${ }^{35}$ followed by lyophilization.

Protein preparation. The fusion proteins were cleaved with trypsin (Promega) in $25 \mathrm{mM}$ Tris- $\mathrm{HCl}-150 \mathrm{mM}$ $\mathrm{NaCl}$ buffer $\mathrm{pH} 7$ for 2 hours to yield DCBMs. The trypsin digestion reaction was followed by RP-UPLC using a $2.1 \times 100 \mathrm{~mm}, 1.7 \mu \mathrm{m}, \mathrm{C} 4 \mathrm{BEH} 300 \mathrm{prST}$ column and an Acquity I-Class system (Waters, MA, USA). The proteins were eluted by a gradient from $20-60 \%$ water to acetonitrile, both containing $0.1 \%$ trifluoroacetic acid. Concentrations were determined using standard samples determined by amino acid analysis (Uppsala University, Sweden).

The HFBI-DCBM-12 was cleaved using papain (Sigma), in $0.1 \mathrm{mM}$ Sodium Phosphate buffer $\mathrm{pH} 7$ for o/n in room temperature to yield CBM-TrCel7A and CBM-TrCel6A. The identity of CBMs were verified by amino acid analysis and MALDI-TOF MS (Autoflex II).

Protein labelling. Proteins were labelled with tritium by reductive methylation ${ }^{7,20,27} .1 .9 \mathrm{mg}$ of protein was dissolved in $1.9 \mathrm{~mL}$ of $0.2 \mathrm{M}$ borate buffer, $\mathrm{pH} 8.96$ and cooled on an ice bath. $13.2 \mu \mathrm{L}$ of $0.37 \%$ formaldehyde 
and $100 \mathrm{mCi}$ of ${ }^{3} \mathrm{H}$ enriched $\mathrm{NaBH} 4\left(10 \mathrm{Cimmol}^{-1}\right.$, PerkinElmer) in $150 \mu \mathrm{L}$ of $0.01 \mathrm{M} \mathrm{NaOH}$ were added and reacted for 30 minutes. The reaction was terminated by RP-HPLC. The specific activities were $0.52 \mathrm{Cimmol}^{-1}$, $0.69 \mathrm{Cimmol}^{-1}$ and $1.25 \mathrm{Cimmol}^{-1}$ for DCBM-12, -24 and -48 .

Binding isotherms. Binding studies were done essentially as reported previously ${ }^{20,27,36,37}$. A $100 \mu \mathrm{M}$ stock solution with $10 \%{ }^{3} \mathrm{H}$-labelled proteins in $100 \mathrm{mM}$ sodium acetate buffer ( $\mathrm{pH} 5.0$ ) with $100 \mathrm{mM} \mathrm{NaCl}$ and $0.1 \%$ BSA was prepared. Dilutions were made with the same buffer. 100-200 $\mu \mathrm{L}$ of each protein solution was mixed with an equal volume of $1-2 \mathrm{~g} \mathrm{~L}^{-1} \mathrm{CNF}$ in water (or $1.28 \mathrm{gL}^{-1} \mathrm{BMCC}$ ) and stirred at ambient temperature with $250 \mathrm{rpm}$ for $1 \mathrm{~h}$. The suspensions were then filtered (Millipore, Millex ${ }^{\circledR}-\mathrm{GV}$ filter unit, $0.22 \mu \mathrm{m}$,) and the free protein was determined by liquid scintillation (PerkinElmer, Tri-Carb 2810TR).

Xylanase assay. The pI9 xylanase of T. reesei was used to hydrolyze xylan from $\mathrm{CNF}^{21,38}$. The hydrolysis of CNF was carried out for $24 \mathrm{~h}$ in $45^{\circ} \mathrm{C}$. In a control CNF sample xylanase was ommitted. The hydrolysed and non-hydrolysed CNF was used to study the effect of xylan hydrolysis with CBM-TrCel7A using a $2.5 \mu \mathrm{M}$ stock solution of the $\mathrm{CBM}$ containing $10 \% 3 \mathrm{H}$-labelled protein. The reactions were terminated by filtrating.

Exchange rate assay. Exchange rate experiments were done as previously ${ }^{26,39}$ by competition between ${ }^{3} \mathrm{H}$-labeled and unlabeled protein. A $100 \mu \mathrm{L}$ aliquot of BMCC (1.28 g L-1) or CNF $(2 \mathrm{~g} \mathrm{~L}-1)$ was mixed with $100 \mu$ $\mathrm{L}^{3} \mathrm{H}$-labelled protein. After binding, the same amount of unlabeled protein was added. The concentration of the added protein was the same as that of the free protein in the equilibrium reaction. The experiments were carried out by taking multiple time points from a set of parallel samples.

Calculations used for partitioning coefficient and Gibb's free energy difference. Langmuir one-site binding model is written in the equation (3) below.

$$
B=\frac{\left[B_{\max }\right] \times[\text { Free }]}{K_{d} \times[\text { Free }]},
$$

where $B_{\max }$ is the maximum binding capacity, $K_{d}$ is the dissociation constant, and [Free] is the concentration of the free protein. When the free protein concentration approaches zero the initial slope of the curve is described by equation (4),

$$
\lim _{\text {Free } \rightarrow 0}\left(\frac{d B}{d \text { Free }}\right)=\frac{\left[B_{\text {max }}\right]}{K_{d}}=K_{r}
$$

where $\mathrm{K}_{\mathrm{r}}$ is the partitioning coefficient and describes the relation of adsorption and desorption at very low protein concentrations.

Gibb's free energy is (equation (5)),

$$
\Delta G=R T \ln \left(K_{d}\right),
$$

and using equation (1) it can be written as show in equation (6),

$$
\Delta G=R T\left(\ln \left(B_{\max }\right)-\ln \left(K_{r}\right)\right)
$$

where $\mathrm{R}$ is the universal gas constant and $\mathrm{T}$ is temperature.

\section{References}

1. Henrissat, B. Cellulases and their interaction with cellulose. Cellulose 1, 169-196 (1994)

2. Várnai, A. et al. Carbohydrate-Binding Modules of Fungal Cellulases. Occurrence in Nature, Function, and Relevance in Industrial Biomass Conversion. Adv. Appl. Microbiol. 88, 103-165 (2014).

3. Hägglund, P. et al. A cellulose-binding module of the Trichoderma reesei $\beta$-mannanase Man $5 \mathrm{~A}$ increases the mannan-hydrolysis of complex substrates. J. Biotechnol. 101, 37-48 (2003).

4. Raghothama, S. et al. Solution structure of the CBM10 cellulose binding module from pseudomonas xylanase A. Biochemistry 39, 978-984 (2000)

5. Stern, D. L. The genetic causes of convergent evolution. Nat. Rev. Genet. 14, 751-764 (2013).

6. Conant, G. C. \& Wagner, A. Convergent evolution of gene circuits. Nat. Genet. 34, 264-266 (2003).

7. Linder, M., Salovuori, I., Ruohonen, L. \& Teeri, T. T. Characterization of a Double Cellulose-binding Domain. J. Biol. Chem. 271, 21268-21272 (1996).

8. Linder, M. \& Teeri, T. T. The roles and function of cellulose-binding domains. Journal of Biotechnology 57, 15-28 (1997).

9. Tomme, P. \& Warren, R. Cellulose-binding domains: classification and properties. ACS Symposium Series, American Chemical Society. doi: 10.1021/bk-1995-0618 (1995).

10. Viikari, L., Vehmaanperä, J. \& Koivula, A. Lignocellulosic ethanol: From science to industry. Biomass and Bioenergy 46, 13-24 (2012).

11. Wilson, D. B. Cellulases and biofuels. Current Opinion in Biotechnology 20, 295-299 (2009).

12. Linder, M. et al. Identification of functionally important amino acids in the cellulose-binding domain of Trichoderma reesei cellobiohydrolase I. Protein Sci. 4, 1056-1064 (1995).

13. Daly, N. L. \& Craik, D. J. Structural studies of conotoxins. IUBMB Life 61, 144-150 (2009).

14. Linder, M., Lindeberg, G., Reinikainen, T., Teeri, T. T. \& Pettersson, G. The difference in affinity between two fungal cellulosebinding domains is dominated by a single amino acid substitution. FEBS Lett. 372, 96-98 (1995).

15. Lehtiö, J. et al. The binding specificity and affinity determinants of family 1 and family 3 cellulose binding modules. Proc. Natl. Acad. Sci. USA 100, 484-489 (2003).

16. Igarashi, K. et al. Traffic jams reduce hydrolytic efficiency of cellulase on cellulose surface. Science 333, 1279-1282 (2011). 
17. Igarashi, K. et al. High speed atomic force microscopy visualizes processive movement of Trichoderma reesei cellobiohydrolase I on crystalline cellulose. J. Biol. Chem. 284, 36186-36190 (2009).

18. Mammen, M., Choi, S.-K. \& Whitesides, G. M. Polyvalent Interactions in Biological Systems: Implications for Design and Use of Multivalent Ligands and Inhibitors. Angew. Chemie Int. Ed. 37, 2754-2794 (1998).

19. Malho, J.-M. et al. Modular Architecture of Proteins Binding Units for Designing Properties in Cellulose Nanomaterials. Angew. Chemie Int. Ed. Accepted (2015).

20. Carrard, G. \& Linder, M. Widely different off rates of two closely related cellulose-binding domains from Trichoderma reesei. Eur. J. Biochem. 262, 637-643 (1999).

21. Arola, S., Malho, J., Laaksonen, P., Lille, M. \& Linder, M. B. The role of hemicellulose in nanofibrillated cellulose networks. Soft Matter 9, 1319 (2013).

22. Kraulis, J. et al. Determination of the three-dimensional solution structure of the C-terminal domain of cellobiohydrolase I from Trichoderma reesei. A study using nuclear magnetic resonance and hybrid distance geometry-dynamical simulated annealing. Biochemistry 28, 7241-7257 (1989).

23. Beckham, G. T. et al. The O-Glycosylated linker from the Trichoderma reesei family 7 cellulase is a flexible, disordered protein. Biophys. J. 99, 3773-3781 (2010).

24. Teeri, T. T., Lehtovaara, P., Kauppinen, S., Salovuori, I. \& Knowles, J. Homologous domains in Trichoderma reesei cellulolytic enzymes: Gene sequence and expression of cellobiohydrolase II. Gene 51, 43-52 (1987).

25. Fox, J. M. et al. A single-molecule analysis reveals morphological targets for cellulase synergy. Nat. Chem. Biol. 9, 356-361 (2013).

26. Linder, M. \& Teeri, T. T. The cellulose-binding domain of the major cellobiohydrolase of Trichoderma reesei exhibits true reversibility and a high exchange rate on crystalline cellulose. Proc. Natl. Acad. Sci. USA 93, 12251-12255 (1996).

27. Varjonen, S. et al. Self-assembly of cellulose nanofibrils by genetically engineered fusion proteins. Soft Matter 7, 2402 (2011).

28. Várnai, A., Huikko, L., Pere, J., Siika-aho, M. \& Viikari, L. Synergistic action of xylanase and mannanase improves the total hydrolysis of softwood. Bioresour. Technol. 102, 9096-9104 (2011).

29. Wada, M., Sugiyama, J. \& Okano, T. Native celluloses on the basis of two crystalline phase ( $\mathrm{I} \alpha / \mathrm{I} \beta)$ system. J. Appl. Polym. Sci. 49, 1491-1496 (1993).

30. Czaja, W., Romanovicz, D. \& Brown, R. malcol. Structural investigations of microbial cellulose produced in stationary and agitated culture. Cellulose 11, 403-411 (2004).

31. Pääkko, M. et al. Enzymatic hydrolysis combined with mechanical shearing and high-pressure homogenization for nanoscale cellulose fibrils and strong gels. Biomacromolecules 8, 1934-1941 (2007).

32. Happs, R. M. et al. O-glycosylation effects on family 1 carbohydrate-binding module solution structures. FEBS J. doi: 10.1111/ febs.13500 (2015)

33. Chen, L. et al. Specificity of O-glycosylation in enhancing the stability and cellulose binding affinity of Family 1 carbohydratebinding modules. Proc. Natl. Acad. Sci. USA 111, 7612-7617 (2014).

34. Swerin, A., Odberg, L., Lindstrbm, T. \& Pulp, S. Deswelling of hardwood kraft pulp fibers by cationic polymers. Nord. Pulp Pap. Res. J. 5, 188-196 (1990).

35. Linder, M. et al. The hydrophobins HFBI and HFBII from Trichoderma reesei showing efficient interactions with nonionic surfactants in aqueous two-phase systems. Biomacromolecules 2, 511-517 (2001).

36. Linder, M. \& Teeri, T. T. The cellulose-binding domain of the major cellobiohydrolase of Trichoderma reesei exhibits true reversibility and a high exchange rate on crystalline cellulose. Proc. Natl. Acad. Sci. USA 93, 12251-12255 (1996).

37. Palonen, H., Tenkanen, M. \& Linder, M. Dynamic interaction of Trichoderma reesei cellobiohydrolases Cel6A and Cel7A and cellulose at equilibrium and during hydrolysis. Appl. Environ. Microbiol. 65, 5229-5233 (1999).

38. Tenkanen, M., Puls, J. \& Poutanen, K. Two major xylanases of Trichoderma reesei. Enzyme Microb. Technol. 14, 566-574 (1992).

39. Carrard, G. \& Linder, M. Widely different off rates of two closely related cellulose-binding domains from Trichoderma reesei. Eur. J. Biochem. 262, 637-643 (1999).

\section{Acknowledgements}

This work was funded by The Academy of Finland and The National Graduate School for Biomass Refining. This work was carried out under the Academy of Finland's Centre of Excellence Program (2014-2019) and project \# 259034 from the Academy of Finland. Riitta Suihkonen is acknowledged for the technical assistance in protein production and purification.

\section{Author Contributions}

S.A. planed and conducted the experimental work, analyzed the results, prepared figures, and wrote the manuscript. M.B.L. reviewed the manuscript and supervised the work.

Additional Information

Supplementary information accompanies this paper at http://www.nature.com/srep

Competing financial interests: The authors declare no competing financial interests.

How to cite this article: Arola, S. and Linder, M. B. Binding of cellulose binding modules reveal differences between cellulose substrates. Sci. Rep. 6, 35358; doi: 10.1038/srep35358 (2016).

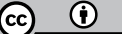

This work is licensed under a Creative Commons Attribution 4.0 International License. The images or other third party material in this article are included in the article's Creative Commons license, unless indicated otherwise in the credit line; if the material is not included under the Creative Commons license, users will need to obtain permission from the license holder to reproduce the material. To view a copy of this license, visit http://creativecommons.org/licenses/by/4.0/

(c) The Author(s) 2016 